\title{
CONSIDERAÇÕES SOBRE CULTURA, ESPAÇO E GEOGRAFIA RURAL
}

\author{
Maryna Vieira Martins Antunes
}

Universidade Estadual Paulista - UNESP, Programa de Pós-Graduação em Geografia, Presidente Prudente, SP. CAPES/Proex

\section{RESUMO}

O objetivo do artigo é sistematizar os apontamentos sobre as articulações entre o conceito de cultura e a espacialidade humana. Realizado a partir de uma pesquisa bibliográfica, o texto centra-se nas contribuições dos Estudos Culturais para a Geografia, com foco na Geografia Rural e aponta que, a despeito do crescente interesse e papel decisivo que a cultura ganha no período atual, estudos com essa abordagem ainda são pouco difundidos na Geografia brasileira, especialmente em relação às questões envolvendo o espaço rural.

Palavras-chave: Cultura; geografia rural; estudos culturais.

\section{CONSIDERATIONS ON CULTURE, SPACE AND RURAL GEOGRAPHY}

\begin{abstract}
The purpose of this article is to systematize the notes about the articulations between the concept of culture and human spatiality. Based on a bibliographical research, the text focuses on the contributions of Cultural Studies to Geography, focusing on Rural Geography and points out that, in spite of the increasing interest and decisive role that culture has gained in the current period, studies with this approach are still not widespread in Brazilian Geography, especially in relation to issues involving rural areas.
\end{abstract}

Keywords: culture; rural geography; cultural studies.

\section{INTRODUÇÃO}

As mudanças nas ciências humanas e
sociais ocorridas pós-1970 são marcos fundamentais para a valorização e incorporação da cultura como conceito explicativo da sociedade, o que vem a influenciar as investigações no campo da Geografia Cultural. Na Geografia Rural nota-se a necessidade de ampliar o olhar sobre os espaços rurais superandos visões dicotômicas em relação aos espaços urbanos, análises economicistas e reducionistas no que diz respeito à vida no campo e maior foco nos sujeitos que constroem suas espacialidades nesses espaços.

Neste texto, pretendemos sistematizar os apontamentos sobre as articulações entre o conceito de cultura e a espacialidade humana. Para tanto, nos focamos nas contribuições dos Estudos Culturais para a Geografia, com foco na Geografia Rural. O artigo está dividido em duas partes, com exceção desta introdução, metodologia e das considerações finais. $\mathrm{Na}$ primeira parte apresentamos um breve histórico da Geografia Cultural e expomos as influências das novas conceituações de cultura e do desenvolvimento dos Estudos Culturais contemporâneos para a Geografia. Na segunda seção nos preocupamos em apresentar algumas considerações sobre a Geografia Rural, com foco nos estudos brasileiros, e as potencialidades da análise cultural para esse campo.

\section{METODOLOGIA}

O artigo foi elaborado a partir do levantamento bibliográfico de autores e autoras que debatem o tema ou questões relacionadas ao objeto de pesquisa, ou seja, autores clássicos e contemporâneos da Geografia Cultural, Geografia Rural, assim como, dos Estudos Culturais, que envolvem as Ciências Humanas de maneira geral, sobretudo, a área da Antropologia e da Sociologia. 


\section{Geografia, Cultura e a Influência dos Estudos Culturais}

A partir da afirmação de Paul Claval (2002, p. 19) de que "[...] o papel da geografia cultural na atualidade é mais importante do que no passado [...]" é possível tecer algumas considerações sobre o novo caráter que a cultura tem ganhado nos estudos geográficos bem como sobre o crescente interesse de outras ciências sociais pelo espaço.

Antes disso, é válido ressaltar que a geografia, de um modo ou de outro, sempre se interessou por questões culturais. Corrêa \& Rosendahl (2003), citando Claval (1999), indicam dois grandes períodos do desenvolvimento da Geografia Cultural: o primeiro compreendendo desde a institucionalização da Geografia como disciplina no século XIX até a década de 1970 e o segundo a partir desta data, sendo entendido como um período de renovação. Os autores ressaltam que a dimensão cultural da sociedade estava presente nas análises geográficas desde o final do século XIX, sendo a paisagem cultural entendida como o resultado da ação humana alterando a paisagem natural.

Para Claval (2002) os geógrafos do final do século XIX até os anos 1950 estudavam a cultura por um ponto de vista positivista ou naturalista, de modo que, dimensões subjetivas não eram apreciadas, privilegiando-se aspectos materiais como técnicas, paisagens e gêneros de vida. O geógrafo explica que a grande contribuição desse período foi evidenciar os aspectos culturais fundamentais para os estudos geográficos, estes inseridos em três domínios:

a) das relações homens/meio ambiente, através do estudo do meio humanizado, da paisagem, das técnicas e das densidades; b) das relações sociais, a partir do estudo das instituições, da comunicação e da difusão das ideias e das técnicas; c) da organização regional e do papel dos lugares (CLAVAL, 2002, p.19).

Já no século XX a denominada Escola de Berkeley (1925-1975) ganha grande projeção nos estudos de Geografia Cultural, devido, sobretudo, ao trabalho de Carl Sauer (1889-1975). Marcada pelo historicismo, a Geografia Cultural de Sauer se centrava em comunidades tradicionais e na dimensão material da cultura, compreendida como uma entidade supra orgânica (CORRÊA; ROSENDAHL, 2003).

De acordo com Claval (2002), entre os anos de 1960 e 1970, houve ainda por parte dos estudiosos da Geografia Cultural uma tentativa de se alinhar à "Nova Geografia" no sentido de definir uma sistematização metodológica dentro dos parâmetros de tal escola. Entretanto, é somente após os anos de 1970 que a Geografia Cultural deixa de ser tratada como um subdomínio da Geografia Humana e se firma em um patamar mais elevado (CLAVAL, 2002).

Corrêa (2003) pontua que no processo de renovação da Geografia Cultural (1970-80) ocorreu uma reinterpretação da cultura pelos geógrafos. Desse modo, para compreender tamanha mudança neste campo, é necessário levar em conta além do interesse da geografia pelas questões culturais, bem como as implicações disto na investigação geográfica e também como se têm conceituado "cultura", questão esta que se estendeu para as demais ciências humanas.

Antes do processo de renovação da Geografia Cultural a cultura era entendida como um componente da sociedade, a qual possuía uma base maior, material ou econômica (de acordo com a perspectiva teórica). Sendo assim, a interpretação cultural se configurava como uma leitura possível dentre outras (geomorfológica, econômica, social, por exemplo), mas sem caráter explicativo quando desvinculada da base. A partir das influências do pensamento pósmoderno e das contribuições do desenvolvimento das pesquisas sobre cultura, sobretudo, da antropologia, a Geografia Cultural amplia a leitura da cultura para além dos aspectos materiais e também reconhecendo seu papel central para a leitura social. Como Zanatta (2008) explica com a renovação da Geografia Cultural:

Não se tratava mais de estudar a diversidade cultural com base nos seus conteúdos materiais, mas de admitir que a cultura está intimamente ligada ao sistema de representações, de significados, de valores que criam uma identidade que se manifesta mediante construções 


\section{compartilhadas}

socialmente e expressas espacialmente, ou seja, de admitir que a cultura no seu sentido antropológico mais amplo representa todo o modo de vida de uma sociedade, o que não inclui somente a produção de objetos materiais, mas um sistema cultural (valores morais, éticos, hábitos e significados expressos nas práticas sociais), um sistema simbólico (mitos e ritos unificadores) e um sistema imaginário, que serve de liame aos dois últimos, constituindo-se no locus da construção da identidade espacial de um grupo (ZANATTA, 2008, p. 06).

É interessante ressaltar que esse processo se deu no contexto de valorização da cultura em todos os campos das ciências sociais e humanas, impulsionado, sobretudo, pela antropologia, como já citado, numa época de constantes debates políticos e culturais e mudanças sociais, denominado de "Virada Cultural". Como Stuart Hall (1997) explica a virada cultural refere-se "[...] a uma abordagem da análise social contemporânea que passou a ver a cultura como uma condição constitutiva da vida social [...]", no lugar "[...] de uma variável dependente, provocando, assim, nos últimos anos, uma mudança de paradigma nas ciências sociais e nas humanidades (HALL, 1997, p. 09).

Assim, a partir dos anos 1980 esse movimento acarreta o que se denomina de "centralidade da cultura": A expressão "centralidade da cultura" indica aqui a forma como a cultura penetra em cada recanto da vida social contemporânea, fazendo proliferar ambientes secundários, mediando tudo (HALL,1997, p.05). Nesse contexto surge um campo de estudos multidisciplinar denominado de Estudos Culturais, cuja marca característica é o enfoque na dimensão cultural contemporânea. Cita-se como principais contribuições para esse campo o teórico Raymond Williams (1921-1988) e o trabalho do Centro de Estudos Culturais Contemporâneos (CCCS - Centre for Contemporary Cultural Studies) da Universidade de Birmingham liderado por Stuart Hall (19322014) (ESCOSTEGUY, 2001; COSGROVE;JACKSON, 2003).

Como Escosteguy (2001) explica a grande contribuição do CCCS para o entendimento da cultura se dá em dois sentidos: o primeiro é que a cultura se manifesta em qualquer formação social ou época histórica, não se configurando como uma entidade monolítica ou homogênea, o segundo é que a cultura não é apenas um conhecimento ou experiência recebido de uma forma passiva, mas um grande número de intervenções ativas, que são expressas mais notavelmente por meio do discurso e da representação. A autora complementa ressaltando que esse grupo de pesquisadores analisa as "[...]práticas culturais simultaneamente como formas materiais e simbólicas. Logo, postula-se que a criação cultural se situa no espaço social e econômico, dentro do qual a atividade criativa é condicionada" (ESCOSTEGUY, 2001, p. 160).

$\mathrm{Na}$ Geografia é interessante considerar a possibilidade de compreender as estratégias de resistência desenvolvidas por grupos subordinados, demonstrando que as culturas são contestadas politicamente, bem como, de analisar o espaço para além de um mero reflexo de forças históricas que o moldaram, uma vez que "[...] a estrutura espacial é parte ativa da constituição histórica das formas culturais" (COSGROVE; JACKSON, 2003, p. 142).

Cosgrove e Jackson (2003) indicam que os Estudos Culturais influenciaram não somente a Geografia Cultural, mas quase todos os campos da Geografia humana ampliando o interesse pela cultura nas investigações geográficas, expandindo inclusive a reflexão sobre outros conceitos para além da paisagem (conceito chave para a Geografia Cultural). Os autores citam o trabalho de Massey (1984) sobre o processo de reestruturação industrial e sua contribuição para o entendimento do lugar e seus significados nas mudanças espaciais (COSGROVE; JACKSON, 2003).

Ainda que de forma sucinta, é conveniente citar, outro processo de renovação epistemológica nas ciências humanas, datado nos anos 1980, denominado de "Virada Espacial" que pode ser interpretada como uma reorientação metodológica que visa maior atenção aos aspectos espaciais, ou à espacialidade, dos objetos e sujeitos de análise das humanidades. 
Na Geografia temos na obra de Edward Soja ${ }^{1}$ um dos principais geógrafos que teorizou tal movimento, sobretudo no que diz respeito à crítica ao historicismo (FRANK, 2009). Entretanto, como Corrêa (2011) adverte:

A espacialidade humana tem uma essencial centralidade na geografia, constituindo-se na característica distintiva face às outras ciências. Assim, falar em "spatial turn" (virada espacial) em relação à geografia, tal como ocorreu nas outras ciências sociais durante a década de 1980, constituise em equívoco. Razão do ser da geografia a espacialidade pode ser considerada em qualquer escala do espaço geográfico. Mais importante ainda, a espacialidade está presente em todas as matrizes intelectuais que, da segunda metade do século XIX aos dias atuais, marcaram a trajetória geográfica (CORRÊA, 2011, p. 62).

Retomando a afirmação de Claval (2002) é possível perceber como a Geografia Cultural torna-se ainda mais importante no debate contemporâneo sobre a sociedade, uma vez que, no atual contexto as questões que envolvem cultura e espaço são cada vez mais visadas nas ciências sociais. Na seção a seguir procuraremos expor alguns tópicos selecionados para a discussão sobre a influência dos Estudos Culturais nas análises sobre os espaços rurais.

\section{Geografia Rural e os Estudos Culturais}

Ao longo de seu desenvolvimento a Geografia estudou o campo e o rural - este lido como o modo de vida específico do campo - de modo geral, sob três perspectivas: agrícola, agrária ou rural. A Geografia agrícola se preocupava em caracterizar o campo, analisando as técnicas de produção e as estruturas do setor produtivo. No Brasil essa tendência é

\footnotetext{
${ }^{1}$ SOJA, E. W. Geografias Pós-Modernas: a reafirmação da teoria social crítica. Tradução: Vera Ribeiro. Rio de Janeiro: Jorge Zahar, 1993.
}

representada pelos estudos do Instituto Brasileiro de Geografia e Estatística (IBGE) no fim da primeira metade do século XX. Esses estudos se caracterizavam pela escolha de uma determinada área na qual se esgotava a investigação sobre o sistema produtivo, condições do solo, clima, relevo e técnicas de produção (ANDRADE, 1995).

Já na década de 1950 no Brasil os estudiosos começam a investigar as questões políticas e sociais do campo. Autores como Orlando Valverde (1917-2006) e Manuel Corrêa de Andrade (1922-2007) ao lançarem olhar sobre os problemas sociais, estrutura agrária e relações de trabalho no campo contribuíram para o desenvolvimento de uma Geografia Agrária. Esses estudos, por sua vez, ajudam a ampliar o entendimento sobre o campo ao evidenciar que as atividades desenvolvidas no campo não se referiam apenas à agricultura. A fim de aprofundar tais análises a Geografia Rural, sem deixar de explorar as questões agrícolas e agrárias, procura explorar o campo por um viés mais totalizante, abrindo espaço para diferentes tipos de investigação (ANDRADE, 1995).

Santos (2012) argumenta que as concepções sobre espaço rural brasileiro, cunhadas por grande parte da comunidade científica, levaram à construção de uma imagem sobre o campo relacionada ao atraso, ao não moderno. Para o autor os valores culturais, as religiões, a arte desenvolvida nesse espaço foram consideradas como entraves para a modernização, nesse sentido, o espaço rural é analisado em oposição ao espaço urbano. Para Bagli (2006), embora as diferenças entre campo e cidade datem a origem dos espaços urbanos, tais diferenças foram hierarquizadas, construindo-se uma oposição:

Eis o problema: a comparação. Quando grandezas heterólogas são comparadas tendo por base a unilateralidade, equívocos são cometidos. O campo foi olhado tendo como parâmetro uma lógica que não the é peculiar: a lógica das cidades. Sua originalidade foi submetida a uma outra realidade. Destarte, a essência constitutiva da lógica do campo foi suprimida pela aparência das cidades. Mostrou-se 
uma face, ocultando a outra (BAGLI, 2006, p. 02).

A dicotomia entre campo e cidade carrega em si as concepções da ciência moderna, caracterizada pela construção de oposições, como, sujeito e objeto, homem e natureza. Oposições que exprimem "uma tendência a transformar a diferença em hierarquia, em superior e inferior" (GONÇALVES, 2011, p. 41). Como Cosgrove (1998) argumenta, citando Sahlins (1976), nenhum grupo além da própria sociedade burguesa considera seu mundo vivido como cultura, da mesma forma essa sociedade, que é urbana, produziu a ciência moderna que garantia uma posição de superioridade de sua própria cultura sobre as demais, inclusive sobre $o$ modo de vida rural.

$\mathrm{Na}$ década de 1990 os estudos rurais, influenciados pela Virada Cultural, passam a reconhecer a importância da cultura dentro da ruralidade e para sua conceituação. As conceituações e definições tradicionais de rural foram colocadas à prova indicando a necessidade de superar a ideia de rural atrelada a um espaço físico delimitado racionalmente, uma realidade mensurável e demarcada por diferenças demográficas e sociais quantificáveis (densidade demográfica, atividades econômicas, por exemplo) (GORMAN-MURRAY; DARIAN-SMITH; GIBSON, 2008).

A noção de que a cultura não é algo externo, mas que compõe os sujeitos em sociedade, como argumenta Silva (2000), esteve ausente na Geografia, sendo assim, os geógrafos "[...] poucas vezes questionaram com profundidade a condição existencial dos homens, preocupando-se muito mais com a comparação das diferentes paisagens da terra" (SILVA, 2000, p. 14). Na reflexão sobre o espaço rural o questionamento sobre cultura vem a contribuir, inclusive, para a superação das visões dicotômicas em relação ao espaço urbano.

Primeiramente, os Estudos Culturais problematizaram o "fordismo intelectual" que subdivide a ciência em especialidades, áreas temáticas e disciplinares. Para a análise geográfica tal problematização contribuiu, por exemplo, para o estudo do campo e da cidade para além da simples oposição, como sugere Sposito (2010):

Parece-nos promissora a observação de novas práticas socioespaciais, de novas formas de habitat e de interação social, de novos conteúdos culturais que diferentes sujeitos sociais atribuem aos espaços que vivem, independente de suas qualificações, como urbanos ou rurais, segundo os critérios que vínhamos adotando para essa adjetivação (SPOSITO, 2010, p.129 [grifo da autora]).

No mesmo sentido, outra contribuição dos Estudos Culturais diz respeito à problemática do poder, que envolve a construção de significados e identidades. Nessa perspectiva o conceito de rural está imbuído de significações e sua interpretação está em constante disputa de acordo com o grupo que dele se apropria: o agronegócio, os trabalhadores rurais sem terra, os camponeses, os agricultores familiares, a bancada ruralista e a própria academia, cada grupo constrói sua própria significação que se refere a construções discursivas e culturais bastante complexas. Sendo assim, construir intersecções entre a Geografia Rural e a Geografia Cultural, significa apreender a espacialidade ligada às práticas nas quais esses significados são criados e recriados (CORRÊA, 2011).

\section{CONSIDERAÇÕES FINAIS}

Para finalizar este texto é conveniente retomar nosso objetivo ao escrevê-lo, ou seja, apresentar algumas reflexões sobre as possibilidades de intersecções entre a Geografia Cultural e a Geografia Rural. Nosso intento se deu no sentido de apresentar as potencialidades dos conceitos de cultura e espaço para a análise geográfica dos espaços rurais. Como marco teórico nos propomos a discutir as concepções sobre cultura e Geografia Cultural empreendidas no contexto pós-1970, um período de renovações não somente para a ciência, mas para a sociedade como um todo.

Embora as contribuições de Hall (1997) indiquem que no período atual a cultura torna-se central nas análises sociais, vemos ainda pouco difundidos tais estudos na Geografia brasileira, como já argumentaram Corrêa e Rosendahl (2003), o que torna-se mais um elemento motivador para que se prossiga nos 
questionamentos provocados pelos autores e autoras, especialmente em relação às questões envolvendo o espaço rural, tão heterogêneo, politicamente disputado e marcante na formação da sociedade brasileira.

\section{REFERÊNCIAS}

ANDRADE, M. C. Geografia rural: questões teórico-metodológicas e técnicas. Boletim de Geografia Teorética, Rio Claro, v. 25, n. 49/50, p. 4-14, 1995.

BAGLI, P. Campo e cidade: a construção dos mitos. $2006 . \quad$ Disponível em: <http://www2.fct.unesp.br/nera/publicacoes/Ca mpoecidadeaconstrucaodosmitos.pdf $>$. Acesso em: 06 jan. 2014.

CLAVAL, P. "A volta do cultural" na Geografia. Mercator - Revista de Geografia da UFC, ano 01, n. 01, 2002.

CORRÊA, R. L. Reflexões sobre paradigmas, geografia e contemporaneidade. Revista da ANPEGE, v. 7, n. 1, (esp.), p. 59-65, out. 2011.

CORRÊA, R. L. A Geografia Cultural e o Urbano. In.: CORRÊA, R. L.; ROSENDAHL, Z. (Orgs.) Introdução à Geografia Cultural. Rio de Janeiro: Bertrand Brasil, 2003. p. 167-186.

CORRÊA, R. L.; ROSENDAHL, Z. Geografia Cultural: Introduzindo a Temática, os Textos e uma Agenda. In: CORRÊA, R. L.; ROSENDAHL, Z. (Orgs.) Introdução à Geografia Cultural. Rio de Janeiro: Bertrand Brasil, 2003. p. 09-18.

COSGROVE, D. E. Em direção a uma geografia cultural radical: problemas da teoria. Espaço e cultura, Rio de Janeiro, n. 5, dez. 1998.

COSGROVE, D. E.; JACKSON, P. Novos Rumos da Geografia Cultural. In: CORRÊA, R. L. ROSENDAHL, Z. (Orgs.) Introdução à Geografia Cultural. Rio de Janeiro: Bertrand Brasil, 2003. p. 135-146.

ESCOSTEGUY, A. C. Os Estudos Culturais. In: HOHLFELDT, A.; MARTINO, L. C.; FRANÇA, V. V. (Orgs). Teorias da Comunicação: conceitos, escolas e tendências. Petrópolis, RJ: Vozes, 2001, p. 151-170.

FRANK, M. C. Imaginative Geography as a traveling concept: Foucault, Said and the spatial turn. European Journal of English Studies, v. 13, 1 p. $\quad 61-77, \quad 2009$. https://doi.org/10.1080/13825570802708188

GONÇALVES, C. W. P. Os (des)caminhos do ambiente. 15. ed. São Paulo: Contexto, 2011.

GORMAN-MURRAY, A.; DARIAN-SMITH, K.; GIBSON, C. Scaling the Rural: Reflections on Rural Cultural Studies. Australian Humanities Review, v. 45, p. 01-09, 2008.

HALL, S. A centralidade da cultura: notas sobre as revoluções culturais do nosso tempo. Educação e Realidade, FACEDVUFRGS, v. 22, n. 2, p. 15-46, 1997.

SANTOS, J. R. Os Assentamentos Rurais na abordagem da Geografia Cultural: perspectivas históricas e atuais. In: ENCONTRO NACIONAL DE GEOGRAFIA AGRÁRIA, 21., 2012, Uberlândia. Anais... Uberlândia, 2012, p. 01-15.

SILVA, J. M. Cultura e Territorialidades Urbanas: Uma abordagem da pequena cidade. Revista de História Regional, v. 5, n. 2, p. 9-37, 2000.

SPOSITO, M. E. B. A questão cidade-campo: perspectivas a partir da cidade. In: SPOSITO, M. E. B.; WHITACKER, G. M. Cidade e Campo: relações e contradições entre o urbano e o rural. 2.ed. São Paulo: Expressão Popular, 2010.

SOJA, E. W. Geografias Pós-Modernas: a reafirmação da teoria social crítica. Tradução: Vera Ribeiro. Rio de Janeiro: Jorge Zahar, 1993.

ZANATTA, B. A. A Abordagem Cultural na Geografia. Temporis(ação) (UEG), v.1, p. 249-262, 2008.

Recebido para publicação em 16/08/2018

Aceito em 28/08/2018 